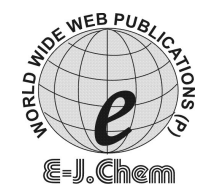

http://www.e-journals.net

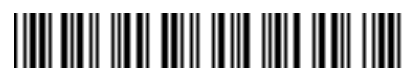

ISSN: 0973-4945; CODEN ECJHAO

E-Journal of Chemistry

Vol. 4, No. 4, pp. 523-530, October 2007

\title{
Photophysical Study of 1,5 - Diaminoanthraquinone in Different Solvents and at Various pH
}

\author{
P. SIVAKUMAR, S. KOTHAI NAYAKI* and M. SWAMINATHAN \\ Department of Chemistry, Faculty of Engg \& Tech., \\ Annamalai University, Annamalainagar- 608002.India. \\ kothaichemish@yahoo.co.in
}

Received 28 February 2007; Accepted 30 March 2007

\begin{abstract}
The absorption and fluorescence spectral properties 1,5-diaminoanthraquinone (DAAQ) have been investigated in a series of organic solvents of different polarity and in aqueous solutions with $\mathrm{H}_{\mathrm{o}} / \mathrm{pH} / \mathrm{H}_{-}$in the range -10 to 17 . The Stokes shifts of DAAQ are correlated with various solvent polarity scales. The better correlation of Stokes shifts of DAAQ with $\mathrm{E}_{\mathrm{T}}(30)$ indicates the predominance of hydrogen bonding interactions over dipolar interactions. The study also reveals that the fluorescence of DAAQ dication is red shifted on protonation. The abnormal fluorescence of DAAQ is found to be due to large solvent relaxation in polar medium. The acidity constants for various prototropic reactions in $\mathrm{S}_{0}$ and $\mathrm{S}_{1}$ states are determined and discussed.
\end{abstract}

Keywords: 1,5-Diaminoanthraquinone, Hydrogen bonding interactions, Solvent relaxation, Excited state acidity constants.

\section{Introduction}

Solvents effects on physical or chemical processes are usually studied by means of empirical parameters ${ }^{1}$, to determine the type of interaction force that predominance in the solvent effect. Among different physicochemical properties of the molecules, the acidity constants are of fundamental importance in planning analytical procedures such as acid-base titrations, extraction and complex formation. The acidity constants of organic molecules are of great importance in the analysis of drugs as well as in the interpretation of their action of mechanism.

Investigations of effect of solvents and $\mathrm{pH}$ on the absorption and fluorescence spectra of amino and hydroxy compounds of fused aromatic and non-fused aromatic systems ${ }^{2-9}$ with one or more functional groups have been studied extensively. 
Anthraquinone derivatives have been widely used in analytical chemistry namely as strong chelating agents and chromophores ${ }^{10,11}$. Diaminoanthraquinones find applications in the fields of biochemistry ${ }^{12}$ and electrochemistry ${ }^{13}$. They are used in the synthesis of electroactive dendrimers ${ }^{14}$ and preparation of solid-state redox super capacitors ${ }^{15}$. In this study we have analysed the spectral characteristics of 1,5-Diaminoanthroquinone (DAAQ) in various solvents and at various $\mathrm{pH}$.

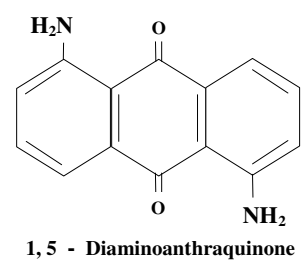

\section{Experimental}

1,5 -Diaminoanthroquinone was obtained from Aldrich Chemical Co and purified by recrystallisation from ethanol. All the solvents used were of highest grade (Spectrograde or AnalaR) commercially available. Triplly distilled water was used for the preparation of aqueous solutions. Solution in the $\mathrm{pH}$ range $1.5-12$ were prepared by adding appropriate amount of $\mathrm{NaOH}$ and $\mathrm{H}_{3} \mathrm{PO}_{4}$. A modified Hammett's acidity scale $\left(\mathrm{H}_{\mathrm{o}}\right)^{16}$ for solutions below pH 1.5 (using $\mathrm{H}_{2} \mathrm{SO}_{4}-\mathrm{H}_{2} \mathrm{O}$ ) and Yagil's basicity scale $\left(\mathrm{H}_{-}\right)^{17}$ for solutions above $\mathrm{pH}$ 12(using $\mathrm{NaOH}-\mathrm{H}_{2} \mathrm{O}$ ) were employed. The concentrations of the solutions were of the order $10^{-4}-10^{-5} \mathrm{M}$. The absorption spectra were recorded using a JASCO UNIDEC7800 spectrophotometer and fluorescence measurements were made using a JASCO FP550 spectrofluorimeter. $\mathrm{pH}$ values in the range 1.5-12.0 were measured on ELICO $\mathrm{pH}$ meter model LI-IOT. The isosbestic wavelengths were used as the excitation wavelength for measuring the fluorescence intensities at any analytical wavelength.

\section{Results and Discussion}

Effect of Solvents

The absorption and fluorescence spectra of DAAQ were recorded in different solvents and these spectral maxmia are complied in Table 1. Relative to cyclohexane, the absorption maxima of DAAQ is red shifted in all the solvents, except water. Compared to cyclohexane the absorption maximum is blue shifted in water. Amino group acts as proton donor in all the solvents except water. In water it behaves as a proton acceptor since proton-donating capability of water is reported to be more. Hence a blue shift relative to cyclohexane is generally observed for all amino compounds in water.

The fluorescence maxima of DAAQ are red shifted from cyclohexane to water. The red shifts observed in hydrogen bonding solvents are due to interaction of solvents with hydrogen atom of the $-\mathrm{NH}_{2}$ group. The absorption and fluorescence solvatochromic shifts reveal that the solvent interactions of hydrogen bonding solvents are predominant. This is confirmed by the correlation of the Stokes shifts of DAAQ with $\mathrm{E}_{\mathrm{T}}(30)^{18}$ and $\mathrm{BK}^{19}$ values (Table 2). The better correlation of Stokes shifts of DAAQ with $\mathrm{E}_{\mathrm{T}}(30)$ indicates predominance of hydrogen bonding interactions over dipolar interactions. The poor correlation value 0.618 for $\mathrm{E}_{\mathrm{T}}(30)$ may be due to the intramolecular hydrogen bonding between $\mathrm{C}=\mathrm{O}$ and $-\mathrm{NH}_{2}$ groups present in the molecule. 
A flat quasi-aromatic ring formed by an intramolecular hydrogen bonding in 1,5dihydroxyanthraqunone ${ }^{20}$ was reported already. So in all the hydrogen bonding solvents a large deviation in Stokes shift is observed.

Table 1. Absorption and fluorescence maxima of 1,5-Diaminoanthroquinone (DAAQ) in different solvents and various $\mathrm{pH}$.

\begin{tabular}{|c|c|c|c|}
\hline No. & Solvents & $\begin{array}{l}\lambda_{\mathrm{abs}} \\
\mathrm{nm}\end{array}$ & $\begin{array}{l}\lambda_{\text {flu }} \\
\mathrm{nm}\end{array}$ \\
\hline $\mathrm{a}$ & Cyclohexane & $\begin{array}{l}269.0 \\
231.1\end{array}$ & 328 \\
\hline b & Dioxane & $\begin{array}{l}282.0 \\
241.3\end{array}$ & 360 \\
\hline $\mathrm{c}$ & Ethyl acetate & $\begin{array}{l}275.0 \\
238.2\end{array}$ & 348 \\
\hline d & Dichloromethane & 276.0 & 340 \\
\hline $\mathrm{e}$ & ter-Butyl alcohol & $\begin{array}{l}278.0 \\
237.1\end{array}$ & 344 \\
\hline$f$ & Acetonitrile & $\begin{array}{l}273.0 \\
233.0\end{array}$ & 335 \\
\hline $\mathrm{g}$ & Isopropyl alcohol & $\begin{array}{l}277.0 \\
236.1\end{array}$ & 345 \\
\hline h & n- Butyl alcohol & $\begin{array}{l}275.0 \\
235.2\end{array}$ & 343 \\
\hline $\mathrm{i}$ & Methanol & $\begin{array}{l}272.0 \\
230.3\end{array}$ & 340 \\
\hline $\mathrm{j}$ & Water & $\begin{array}{l}264.0 \\
227.1\end{array}$ & 353 \\
\hline $\mathrm{k}$ & Dication & $\begin{array}{l}223.0(\mathrm{~s}) \\
248.6 \\
272.8(\mathrm{~s}) \\
328.6(\mathrm{~s})\end{array}$ & 433 \\
\hline 1 & Monoanion & $\begin{array}{l}278.0 \\
232.0\end{array}$ & --- \\
\hline
\end{tabular}

\section{Effect of acid- base concetrations}

The absorption and fluorescence spectra of DAAQ have been studied in the $\mathrm{H}_{\mathrm{o}} / \mathrm{pH} / \mathrm{H}_{-}$ range -10 to 17.0. The relevant data are compiled in Table 1. The absorbance and fluorescence spectra of various phototropic species are given in Figures 1 and 2. When the acidity is increased $(\mathrm{pH}>2)$ the spectrum is blue shifted with the maxima 223(s), 248.6, $272.8(\mathrm{~s})$ and $328.6(\mathrm{~s}) \mathrm{nm}$. This spectrum resembles the spectrum of anthraquinone in water ( $\lambda_{\max }=255,270$ (s) and $323 \mathrm{~nm}$ ). This clearly suggests the direct formation of dication, obtained by the protonation of two amino groups simultaneously. For a symmetrical molecule, simultaneous protonation of both the amino groups are possible. A similar behaviour has been reported in 4,4'-diaminodiphenyl methane ${ }^{21 .}$ 
Table 2. Stokes shifts observed for DAAQ in different solvents with $E_{T}(30)$ and $\mathrm{BK}$ values.

\begin{tabular}{llllc}
\hline No & Solvents & $\mathrm{E}_{\mathrm{T}}(30)$ & $\mathrm{BK}$ & $\Delta \bar{V}\left(\mathrm{~cm}^{-1}\right)$ \\
\hline $\mathrm{a}$ & Cyclohexane & 31.2 & -0.001 & 6687 \\
$\mathrm{~b}$ & Dioxane & 36.0 & 0.043 & 7683 \\
$\mathrm{c}$ & Ethyl acetate & 38.1 & - & 7628 \\
$\mathrm{~d}$ & Dichloromethane & 41.1 & 0.586 & 6820 \\
$\mathrm{e}$ & ter-Butyl alcohol & 43.9 & 0.673 & 6901 \\
$\mathrm{f}$ & Acetonitrile & 46.0 & 0.864 & 6779 \\
$\mathrm{~g}$ & Isopropyl alcohol & 48.6 & 0.766 & 7115 \\
$\mathrm{~h}$ & n- Butyl alcohol & 50.2 & 0.754 & 7209 \\
$\mathrm{i}$ & Methanol & 55.5 & 0.856 & 7353 \\
$\mathrm{j}$ & Water & 63.1 & 0.913 & 9550 \\
& Correlation coefficient & & & \\
& & $\mathrm{E}_{\mathrm{T}}(30)$ & & 0.618 \\
& & $\mathrm{BK}$ & & 0.271 \\
\hline
\end{tabular}

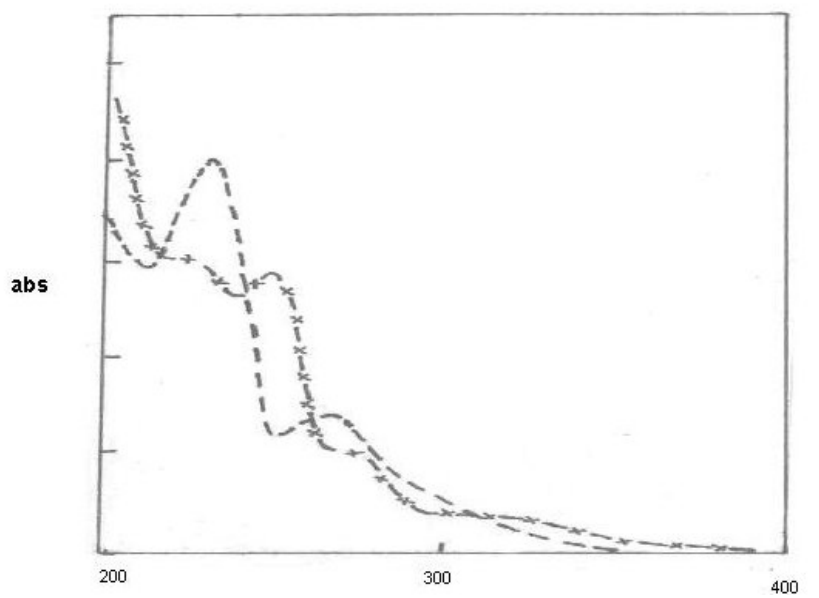

Figure 1. Absorption spectra of different protoropic species of DAAQ.

----- neutral ( $\mathrm{pH} 6) ; \quad-\mathrm{x}-\mathrm{x}-\mathrm{x}-\mathrm{x}-$ dication $(\mathrm{pH}>2)$

The absorption spectra of DAAQ do not change, when the basicity is increased upto H_14.0. But a regular red shift is noticed with further increase in basicity $\left(H_{-}>14.0\right)$. The red shifted spectrum may be due to the formation of monoanion, but the isosbestic point was not constant. This behaviour is generally observed in many aromatic amino compounds. ${ }^{4-7}$. Hence the ground state $\mathrm{pK}_{\mathrm{a}}$ value cannot be determined for neutral-monoanion equilibrium.

The fluorescence characteristics of the cationic species of DAAQ is different from those of absorption. The neutral species shows a spectral maximum of 353.0. Further decrease of acidity below $\mathrm{H}_{0}=0.83$ gives a large red shifted fluorescence spectrum $\left(\lambda_{\max }=433 \mathrm{~nm}\right)$. When the acidity is further increased, there is no significant change in the spectral maximum till $\mathrm{H}_{\mathrm{o}}-10.0$. The excitation spectrum monitored at $433.0 \mathrm{~nm}$ resembles the absorption spectrum of dication. Hence the red shifted peak may be due to dication of DAAQ which is obtained as a result of simultaneous protonation of both the amino groups. 


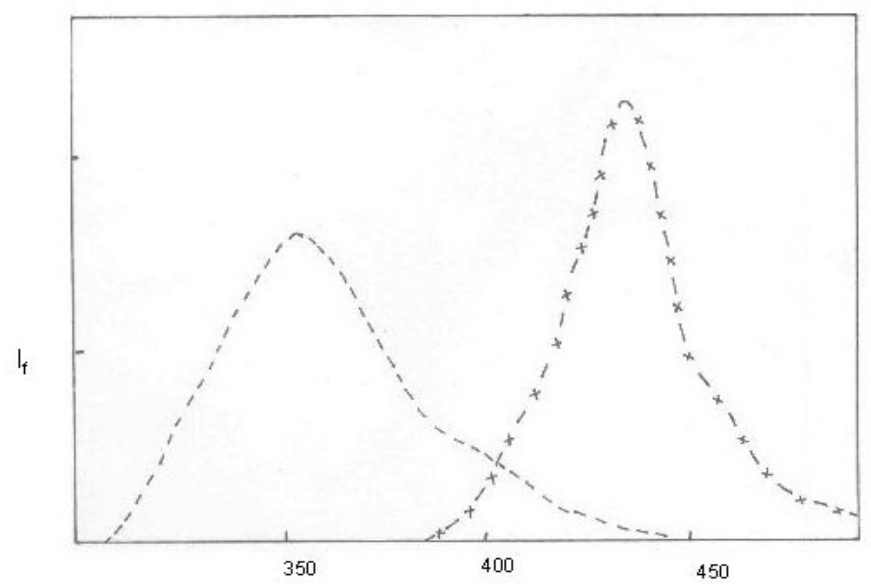

Figure 2. Fluorescence spectra of different species of DAAQ. ------neutrap $(\mathrm{pH}=7)$; - $\mathrm{x}-\mathrm{x}-\mathrm{x}-\mathrm{x}$-dication $\left(\mathrm{H}_{\mathrm{o}}>0.83\right)$

When amino group is protonated usually a blue shift is expected. To find out the reason for the unusual red shift observed for the dication of DAAQ, the fluorescence spectra were recorded in polar and non-polar solvents. The absorption maxima, fluorescence maxima and Stokes shift of neutral and dication of DAAQ in cyclohexane are given in Table 3. The dication in cyclohexane was obtained by the addition of different amounts of trifluoroacetic acid (TFA) in cyclohexane. The absorption spectrum of dication in cyclohexane is matched with those of the species derived by the addition of $\mathrm{H}_{2} \mathrm{SO}_{4}$ in aqueous medium. But the fluorescence spectrum of dication is blue shifted in cyclohexane (Figure 3) and red shifted in water. Hence the unusual red shift in polar solvent like water is due to the large solvent relaxation of dication in the $S_{1}$ state. This is also confirmed by the large Stokes shift for dication in water. In cyclohexane, the Stokes shift of neutral and dicationic species are 6687 and $7189 \mathrm{~cm}^{-1}$ whereas in water they are 9550 and $17131 \mathrm{~cm}^{-1}$ respectively. The large difference in the Stokes shifts of dicationic species in cyclohexane and water must be due to solvent relaxation, because the change in solvent cannot change the vibrational relaxation or a change in energy of the excited state by such a large amount. The large Stokes shift of dication of DAAQ in water also suggests that the dication is more stabilized by polar solvents in the excited state than in the ground state. Similar behaviour was observed for 2,7-diaminofluorene ${ }^{4}$ and 4,4'-diaminobiphenyl ${ }^{6}$. The fluorescence spectrum of dication of DAAQ in cyclohexane has two maxima similar to the fluorescence spectrum of anthraquinone in cyclohexane(300, 340nm).

Table 3. Absorption and fluorescence maxima $(\mathrm{nm})$ and Stokes shift $\left(\mathrm{cm}^{-1}\right)$ of DAAQ in Cyclohexane in presence of TFA.

\begin{tabular}{clcc}
\hline Solvent & $\lambda_{\text {abs }}$ & $\lambda_{\text {flu }}$ & Stokes shift \\
\hline Cyclohexane (neutral) & 269.0 & 328.0 & 6687 \\
& 231.1 & & 7189 \\
Cyclohexane + 1\%TFA & 248.6 & 302.7 & \\
& $273.0(\mathrm{~s}) 327.8$ & & \\
& $328.0(\mathrm{~s})$ & & \\
\hline
\end{tabular}




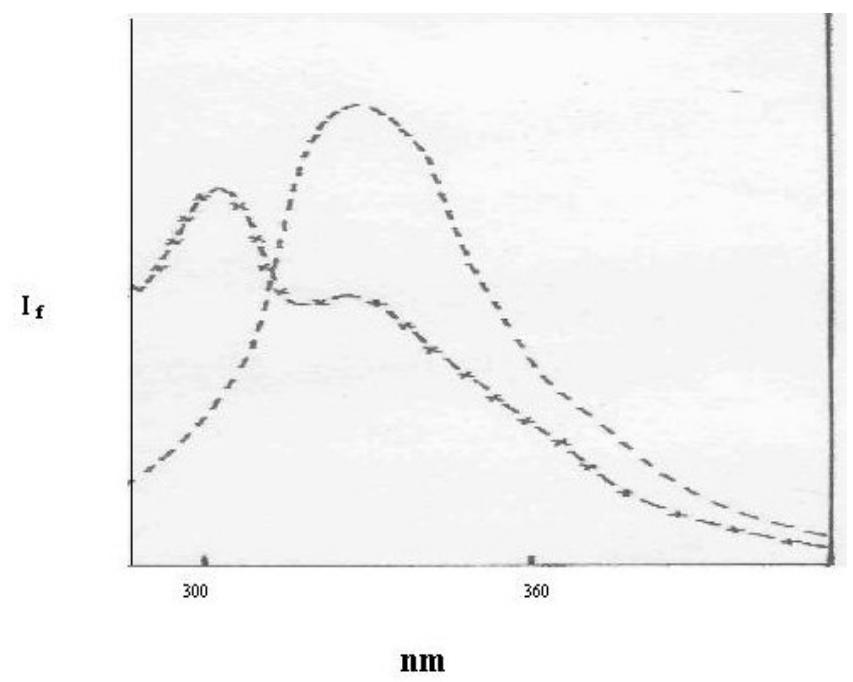

Figure 3. Flurescence spectra of different species of DAAQ in cyclohexane and cyclohexane-TFA medium.

------neutral(cyclohexane); -x-x-x-x dication (cyclohexane-1\% TFA).

When the $\mathrm{pH}$ is increased form 7, the quenching of fluorescence starts in DAAQ and this may be due to the formation of non-fluorescent monoanion. This is consistant with the earlier results that the monoanion formed by the deprotonation of aromatic amines in general are non-fluorescent ${ }^{6,22}$.

\section{Acidity constants}

The acidity constants of DAAQ in ground and excited states are calculated for the following equilibria.

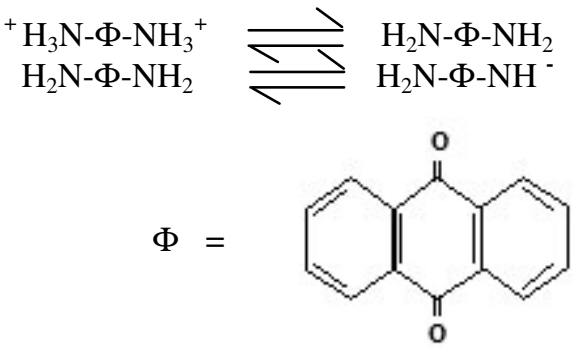

\section{Anthraquinone}

The ground state $\mathrm{pK}_{\mathrm{a}}$ value for dication-neutral equilibrium (1) was calculated spectrophotometrically and listed in the Table 4. The $\mathrm{pK}_{\mathrm{a}}$ value for the neutral-monoanion equilibrium for DAAQ could not be determined because the isosbestic point was not constant.

The $\mathrm{pK}_{\mathrm{a}} *$ values for different proton-transfer reactions of DAAQ were calculated with the help of fluorimetric titration (FT) (Table 4). The FT curves of dication-neutral equilibrium (Figure 4) meet at $\mathrm{Ho}=-0.3$, which is taken to be $\mathrm{pK}_{\mathrm{a}}{ }_{(\mathrm{FT})}$ value. Förster cycle ${ }^{23}$ method was applied to calculate the $\mathrm{pK}_{\mathrm{a}}{ }^{*}$ values for neutral-dication equilibrium. The $\mathrm{pK}_{\mathrm{a}}{ }^{*}$ (abs) value is abnormal due to large blue shift in absorption maximum caused by simultaneous protonation of two amino groups. 
Table 4. $\mathrm{pK}_{\mathrm{a}}$ values of various prototropic equilibria of DAAQ in the lowest excited singlet and ground states.

\begin{tabular}{llllllll}
\hline \multicolumn{2}{c}{ Equilibria } & \multicolumn{3}{c}{ Föster Cycle Method } \\
& $\mathrm{pK}_{\mathrm{a}}$ & $\mathrm{pK}_{\mathrm{a}}{ }^{*}(\mathrm{FT})$ & $\mathrm{pK}_{\mathrm{a}}^{*}$ (abs) & $\mathrm{pK}_{\mathrm{a}}^{*}$ (flu) & $\mathrm{pK}_{\mathrm{a}}^{*}$ (ave) \\
\hline Dication & $\rightarrow$ & Neutral & -0.08 & -0.30 & -11.07 & -4.93 & -8.01 \\
Neutral & $\rightarrow$ & Monoanion & $>14.0$ & 12.3 & -- & -- & -- \\
\hline
\end{tabular}

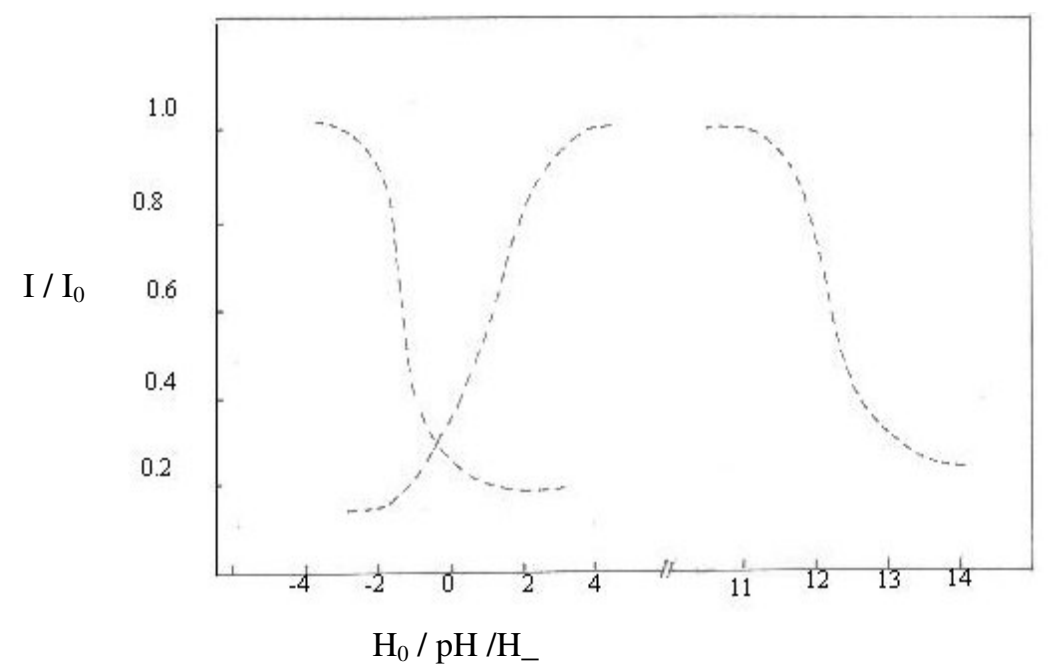

Figure 4. Plot of relative fluorescence intensities (I/Io) Vs $\mathrm{H}_{\mathrm{o}} / \mathrm{pH} / \mathrm{H}-$ of the various prototropic species of DAAQ.

The $\mathrm{pK}_{\mathrm{a}}{ }^{*}$ value for neutral-monoanion equilibrium (2) is determined from the fluorescence quenching curve of the neutral species of DAAQ . The wavelength of constant absorbance was used as $\lambda_{\text {exi }}$ for fluorimetric titration as the isosbestic point was not constant. The $\mathrm{pK}_{\mathrm{a}}{ }^{*}$ value shows that the $-\mathrm{NH}_{2}$ group becomes more acidic in $\mathrm{S}_{1}$ state. Since the monoanion is non-fluorescent, Förster cycle method is not applicable.

\section{References}

1. Reichardt C, Solvents and solvent effects in organic Chemistry, 1990, $2^{\text {nd }}$ Ed. V.C.H, Germany.

2. Tutsumi K and Shisuka H, Z Phys Chem (N.F), 1980 ,122, 129.

3. Swaminathan M and Dogra S K, Can J Chem, 1983, 61, 1064.

4. Monoharan R and Dogra S K, Can J Chem, 1987, 65, 2013.

5. (a) Kothai Nayaki S and Swaminathan M, J Photochem Photobiol A(Chem), 1994, 84, 13. (b) Kothai Nayaki S and Swaminathan M, Spectrochim Acta, Part A. 2002, 58, 2939.

6. Rajendiran N and Swaminathan M, Bull Chem Soc. Jpn. 1995, 68, 2797.

7. Kothai Nayaki S and Swaminathan M, Spectrochim Acta, Part A. 2001, 57, 1361.

8. Rajendiran N and Swaminathan M, Indian J Chem, 1996, 35A, 385.

9. Kothai Nayaki S and Swaminathan M, Spectrochim Acta, Part A, 2006, 64, 631.

10. Navas-Diaz A, Talanta, 1991, 38, 571.

11. Kim I, Kono E and Takagishi T, Textile Reserch Journal, 1996, 66, 763-770. 
12. Gabourdnes M, Bourgine V and Bazin H B, Alpha-Bazin B, Anal Biochem, 2004, 333(1), 105-113.

13. Roullier L, Laviron, E and Waldner E, Electrochem Soc, 2001, 132, 1121-125.

14. George R, Newkome, Venkatraj V, Narayanan, Luis A, Godinez Eduardo PerezCordero and Leis Echegoyen, Macromolecules, 1999, 32, 6782-91.

15. Hashmi S A, Suematsu Shunzo and Naoi Katsuhiko, J Power sources, 2004, 137, 145-151.

16. Jorgenson M J and Hartter D A, J Am Chem Soc, 1963, 85, 878.

17. Yagil G, J Phys Chem, 1967, 17, 1054.

18. (a) Reichart C, Angew. Chem. Int. Ed. Engl, 1979, 18, 98; (b) Reichart C and Dimroth K, Forscher.Chem.Forsch, 1968, 11, 1.

19. Bilot L and Kawski A, Z. Naturforsch,1962, 179, 621.

20. Mario R Reta, Rosa Cattana, Jorge D, Anunziata and Juana J Silber, Spectrochim Acta, Part A, 1993, 49A, 903-912.

21. Rajendiran N and Swaminathan M, Spectrochim. Acta. Part A, 1996, 52, 1785.

22. Rajendiran N and Swaminathan M, J Photochem Photobiol A Chem, 1995, 90, 109.

23. Förster Th, Z Electrochem, 1950, 54, 531. 


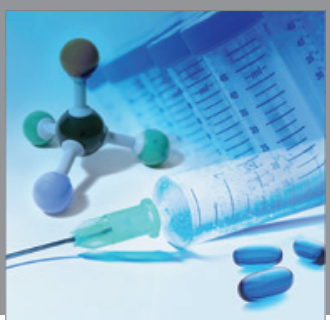

International Journal of

Medicinal Chemistry

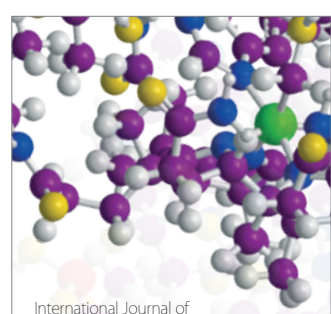

Carbohydrate Chemistry

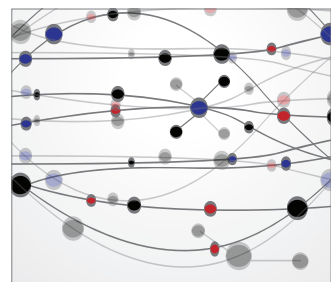

The Scientific World Journal
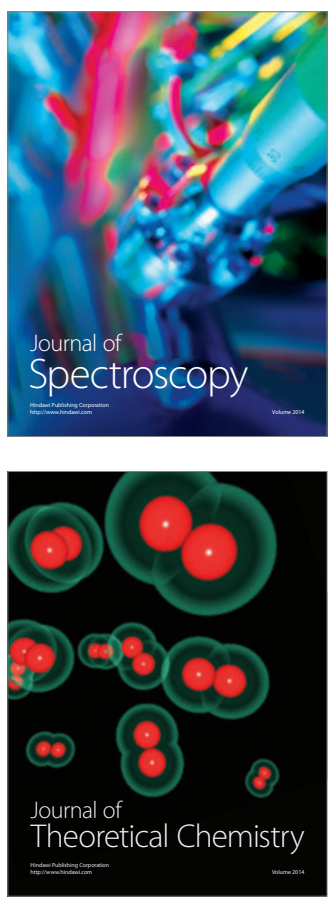
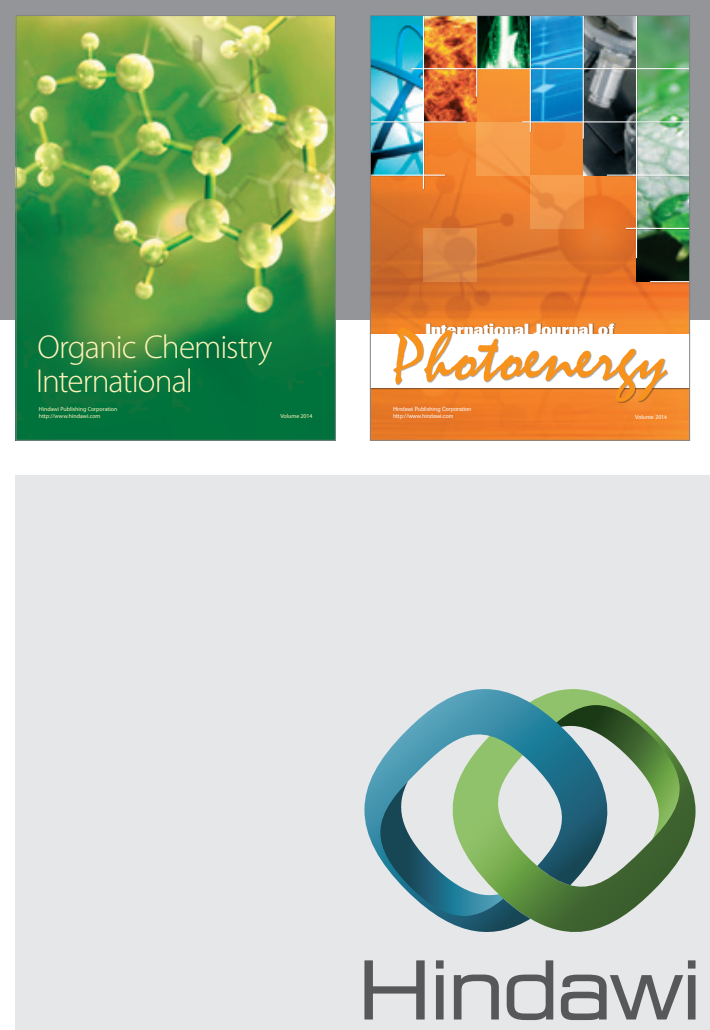

Submit your manuscripts at

http://www.hindawi.com
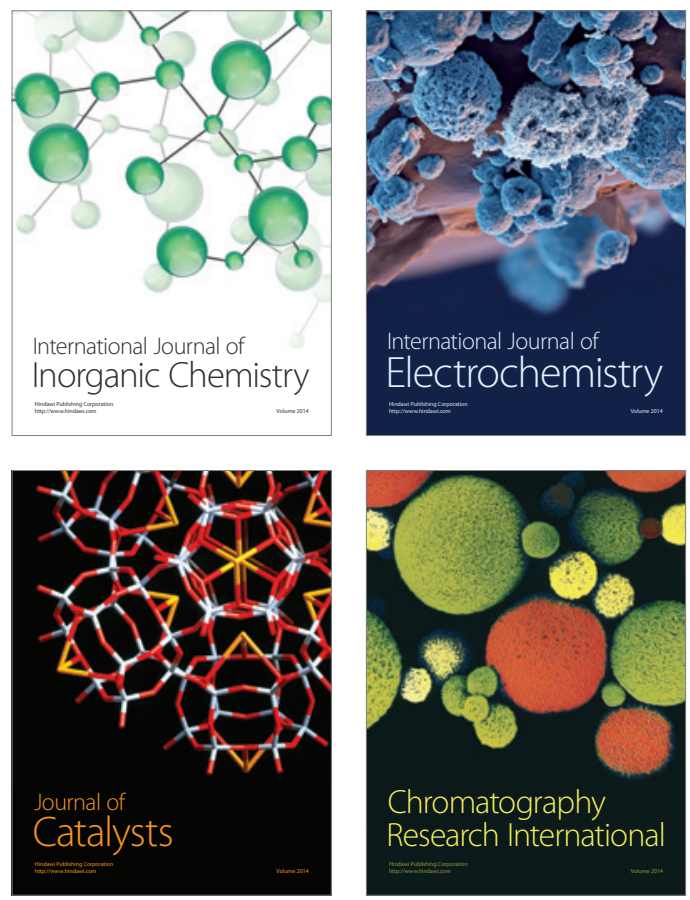
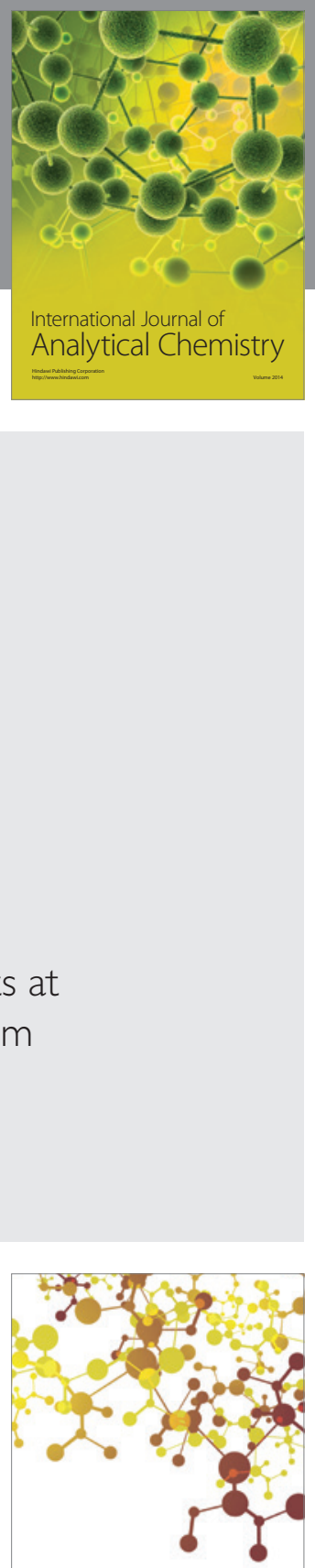

Journal of

Applied Chemistry
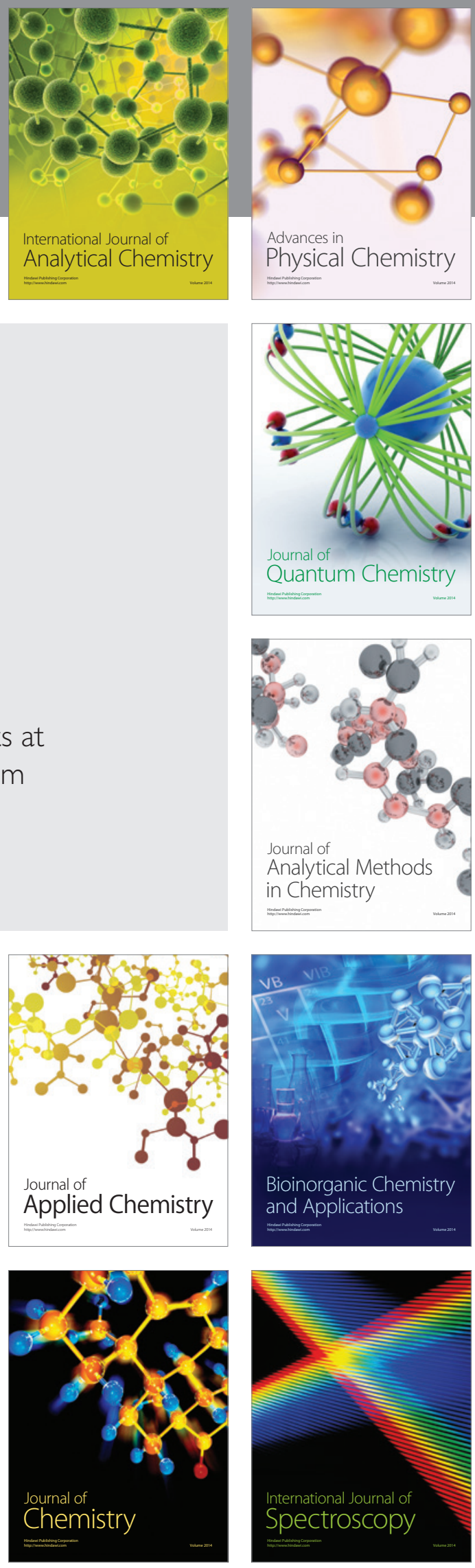